\title{
Perfil axial e radial da massa específica básica da madeira de Miconia cinnamomifolia (DC.) Naudin
}

\author{
Jackson Roberto Eleoterio ${ }^{1 \star}$, Alexander Christian Vibrans ${ }^{1}$, Priscila Kollross ${ }^{2}$, Heitor Felippe Uller ${ }^{1}$, \\ Aline Renata Klitzke ${ }^{1}$
}

\begin{abstract}
${ }^{1}$ Programa de Pós-Graduação em Engenharia Florestal, Universidade Regional de Blumenau, Blumenau, SC, Brasil.
${ }^{2}$ Engenharia Civil, Universidade Regional de Blumenau, Blumenau, SC, Brasil.
\end{abstract}

\begin{abstract}
RESUMO A massa específica é um indicador de qualidade reconhecido pela sua relação com outras propriedades da madeira. Além de a massa específica dar indícios do potencial de uso da madeira, a homogeneidade entre indivíduos e ao longo dos sentidos radial e longitudinal dos troncos está relacionada com a qualidade dos produtos. O objetivo desse trabalho foi determinar a variação da massa específica básica ( $\rho_{\text {bás }}$ ) entre e dentro de indivíduos de Miconia cinnamomifolia (DC.) Naudin (jacatirão-acú). De uma floresta secundária enriquecida com a espécie foram selecionadas nove árvores das quais foram retirados, em média, três discos em diferentes alturas. A $\rho_{\text {bás }}$ foi determinada ao longo de quatro raios por disco. O valor médio foi de $0,581 \mathrm{~g} / \mathrm{cm}^{3}$, com diferença significativa entre árvores. Variações significativas dentro das árvores também foram observadas, relacionadas com o diâmetro à altura do peito sem casca $\left(\mathrm{DAP}_{\mathrm{sc}}\right)$ e com a altura total das árvores, além da posição radial e axial, parâmetros esses significativos em um modelo de regressão. De maneira geral, a massa específica decresce com o aumento do $\mathrm{DAP}_{\mathrm{sc}}$ e com a distância a partir da medula e cresce com a altura a partir da base e com a altura total da árvore.

Palavras-chave: variabilidade; densidade, espécies nativas; qualidade.
\end{abstract}

\section{Axial and radial profile of basic density of Miconia cinnamomifolia (DC.) Naudin wood}

\begin{abstract}
The density is a quality index widely recognized by your relationship with other wood properties. In addition to the density being an indicator of potential use of wood, its homogeneity is related to the quality of the final products. The objective of this work was to determine the variation of the basic density ( $\left.\rho_{\text {bás }}\right)$ between and within trees of Miconia cinnamomifolia (DC.) Naudin (jacatirão-acú). From a secondary forest enriched with the specie were selected nine trees, of which on average three disks were cut at different heights. The $\rho_{\text {bás }}$ was determined along four rays per disc. The mean value was $0.581 \mathrm{~g} / \mathrm{cm}^{3}$, with a significant difference between trees. Significant variations within the trees were also observed, related to the diameter at the breast height under bark $\left(\mathrm{DBH}_{\mathrm{ub}}\right)$ and the total height of the trees, besides the radial and axial position, parameters that are significant in a regression model. In general, the density decreases with increasing DHB and with distance from the pith and grows with the height from the base and with the total height of the tree.
\end{abstract}

Keywords: variability, density, native species, quality.

\section{Introdução}

Algumas espécies florestais secundárias despontam com potencial para o enriquecimento florestal ou para a implantação de plantios florestais homogêneos (SCHWARTZ et al., 2016; RAPPAPORT; MONTAGNINI, 2014; REITZ et al., 1978). Estas espécies poderiam suprir a demanda por madeira de qualidade para construção civil e movelaria, não atendida pelos plantios florestais de Pinus e Eucalyptus. Entre essas espécies está a Miconia cinnamomifolia (DC.) Naudin (jacatirão-açú), árvore perenifólia pertencente à família Melastomataceae, que ocorre naturalmente na Floresta Ombrófila Densa no domínio da Mata Atlântica (GOLDENBERG; CADDAH, 2015). Suas dimensões normalmente estão entre os 15 a 20 m 
de altura e 30 a $40 \mathrm{~cm}$ de diâmetro à altura do peito (DAP) (REITZ et al., 1978).

Nos remanescentes florestais de Floresta Ombrófila Densa do Estado de Santa Catarina a espécie ocupa a nona posição quanto ao valor de importância, com valores médios seguidos de seu intervalo de confiança para densidade de $10,79 \pm 3,6$ ind.ha $a^{-1}$, DAP de 22,1 $\pm 2,23 \mathrm{~cm}$, volume do fuste de $5,13 \pm 2,82 \mathrm{~m}^{3} \cdot \mathrm{ha}^{-1}$ (VIBRANS et al., 2013). A maior parte dos fragmentos florestais catarinenses é formada por florestas secundárias, e estes podem fornecer produtos úteis ao homem além dos importantes serviços ambientais. Sob este contexto, Schuch et al. (2008) e Fantini et al. (2016) relatam a aptidão da espécie para o manejo madeireiro, que pode contribuir para o incremento da renda dos agricultores e para a diversificação na oferta de madeira. Entretanto, o mercado de madeira de espécies das formações secundárias nunca foi desenvolvido, pois por muito tempo houve suficiente suprimento de madeiras nobres e após o esgotamento destas, a oferta de madeiras com baixo preço vindas da Amazônia e a introdução de exóticas cobriram a demanda regional (FANTINI; SIMINSKI, 2005).

Para a introdução de uma espécie em novos mercados, informações técnicas a respeito da madeira são necessárias e a massa específica é amplamente reconhecida como um indicador de qualidade da madeira, associada positivamente com a resistência mecânica (KRETSCHMANN, 2010) e com a condutividade térmica (SIAU, 1982). Mais recentemente, essa propriedade física tem sido intensamente pesquisada com o objetivo de quantificar o acúmulo de carbono em florestas naturais (GOUSSANOU et al., 2016, MATE et al., 2014).

A variabilidade é inversamente relacionada com a qualidade no uso estrutural da madeira. Espécies com grande variação na massa específica entre indivíduos e ao longo dos eixos longitudinal e radial resultam em peças serradas também com grande variabilidade nas propriedades mecânicas, levando à redução dos limites de resistência considerados. A homogeneidade da massa específica é tida como uma vantagem para o uso em produtos de madeira sólida (KNAPIC et al., 2008; ZOBEL; VAN BUIJTENEN, 1989). Quando o objetivo é quantificar o acúmulo de carbono, a variabilidade na massa específica resulta em maiores incertezas nas estimativas.

Em uma ampla revisão sobre a formação do lenho juvenil, especialmente em folhosas, Vidaurre et al. (2011) destacaram que um dos principais indicadores da presença desse tipo de lenho é a menor massa específica e que, para a utilização de madeira sólida, a delimitação da madeira juvenil é muito importante. Em espécies de rápido crescimento como algumas dos gêneros Pinus e Eucalyptus são observadas variações nas propriedades físicas e mecânicas resultantes da presença de lenho juvenil, como registrado por Pelozzi et al. (2012). Estes autores constataram aumento na massa específica do lenho adulto em relação ao juvenil de Eucalyptus grandis Hill ex. Maiden de $26,0 \%$ a 34,9\%, enquanto para Pinus taeda L. essas diferenças são ainda maiores, chegando a 64,5\%. Em Pinus sylvestris L., Fernandes et al. (2017) encontraram tendência de aumento da massa específica no sentido radial de até $18 \%$, padrão comum para espécies heliófilas.

Os efeitos dos fatores genéticos, ambientais e de suas interações sobre a massa específica estão longe de serem elucidadas, entretanto é unanimidade que a maior causa de variação é o genótipo da espécie (BASTIN et al., 2015). O trabalho de Guilley et al. (2004) corrobora com essa ideia, uma vez que foram observadas variações intraespecíficas na massa específica da madeira de Quercus petraea (Matt.) Liebl quando as condições ambientais foram mantidas constantes, possibilitando o entendimento que o agente de variação foi 
genético. Segundo esses autores, a qualidade do sítio afeta o crescimento e não diretamente a massa específica.

Com relação ao efeito que o grau de dominância da árvore exerce na massa específica, Deng et al. (2014) não encontraram relação significativa para Pinus massoniana Lamb., nem Fajardo (2016) para duas espécies de Nothofagus. Uma explicação para essa ausência de relação poderia ser a necessidade de maior resistência para suportar maior peso de copa nos indivíduos dominantes. Dessa forma, a correlação negativa esperada entre o crescimento de indivíduos dominantes e massa específica nem sempre é a regra (SOTELO MONTES et al., 2017).

O perfil radial de variação da massa específica pode ser constante, decrescente ou crescente (WAASENBERG et al., 2015). As espécies tolerantes à sombra podem apresentar um perfil radial constante ou até decrescente, pois no momento em que alcançam os estratos superiores da floresta recebem mais luz, o que resulta em aumento do crescimento (BASTIN et al., 2015). Nock et al. (2009) também relacionam a tolerância à sombra a um perfil radial mais homogêneo. Outro fator que pode determinar a variação na massa específica é a presença de cerne, pois a deposição de extrativos resulta em aumento da massa específica (FAJARDO, 2016).

Diante da falta de informações sobre a variabilidade da massa específica básica de Miconia cinnamomifolia, os objetivos desse trabalho foram: a) identificar se há variação entre árvores e entre posições ao longo dos eixos axial e radial e b) ajustar um modelo de regressão para estimar essa propriedade física.

\section{Material e Métodos}

\section{Área de estudo}

A área de estudo encontra-se no município de Guaramirim - SC (26²'10”S e 4902'38”O), localizada em uma floresta secundária com área de 42 hectares e altitudes que variam de 160 a 500 m. De acordo com Köppen, o clima é do tipo Cfa, subtropical mesotérmico úmido com verão quente e sem estação seca (ALVARES et al., 2013). Os solos predominantes da área de estudo são os Cambissolos háplicos (EMBRABA, 2004). O relevo é fortemente ondulado com declividade variando de 10 a 40\% (SILVA et al., 2015).

A vegetação original do local de estudo é classificada conforme IBGE (2012) como Floresta Ombrófila Densa e de acordo com Oliveira-Filho et al. (2015), como floresta pluvial subtropical. As espécies características dessa formação originalmente eram Sloanea guianensis (Aubl.) Benth., Ocotea catharinensis Mez, Calyptranthes lucida Mart. ex DC., Brosimum lactescens (S.Moore) C.C.Berg, Chrysophyllum viride Mart. \& Eichler, Guapira opposita (Vell.) Reitz, Cryptocarya aschersoniana Mez., além de Euterpe edulis Mart., como espécie dominante do sub-bosque (KLEIN, 1978).

A floresta que compõe a área de estudo é proveniente de um plantio de adensamento realizado no ano de 1978, após um histórico de intensa exploração dessa floresta que deixou como legado pastagens intercaladas com capões de floresta em estágios variados de sucessão. As espécies nativas escolhidas para o adensamento foram Miconia cinnamomifolia, Hieronyma alchorneoides Allemão e canelas do gênero Nectandra spp. Com intuito de amenizar a competição e conter as plantas espontâneas, o proprietário realizou roçadas nos anos iniciais à implantação da floresta (SILVA et al., 2015). Sem posterior intervenção, exceto o corte de palmiteiros (Euterpe edulis) e eventualmente de madeira, as espécies da flora local se estabeleceram, tornando a floresta fisionomicamente semelhante a uma floresta secundária da mesma idade. 


\section{Coleta de dados}

A base de dados consistiu em informações de nove árvores de Miconia cinnamomifolia com as dimensões apresentada na Tabela 1. As alturas de retirada dos discos variaram entre as árvores.

\section{Procedimento experimental}

De cada disco, com espessura nominal de $2,5 \mathrm{~cm}$, foram amostrados quatro raios, sendo o primeiro em ângulo de $45^{\circ}$ ao maior raio e os demais perpendiculares ao anterior. De cada raio foi retirada uma faixa com largura de $1 \mathrm{~cm}$, cortada com serra fita. Cada faixa foi subdividida a cada $1 \mathrm{~cm}$, resultando em elementos com dimensões nominais de $1 \mathrm{~cm} \mathrm{x}$ $1 \mathrm{~cm} \times 2,5 \mathrm{~cm}$, sendo essa última medida no sentido axial.

A determinação da massa específica básica, definida como a razão entre massa anidra e volume saturado iniciou pela saturação dos raios, sob vácuo, na temperatura ambiente, durante 72 h. O volume saturado foi determinado pelo método da balança hidrostática, com resolução de $0,1 \mathrm{mg}$. Para determinação da massa seca, os corpos de prova foram mantidos na estufa a $103 \pm 2^{\circ} \mathrm{C}$ até massa constante, sendo em seguida pesados também com resolução de $0,1 \mathrm{mg}$.

\section{Análise estatística}

A massa específica básica média de cada árvore foi comparada pelo teste de Tukey $(\alpha=5 \%)$ utilizando a função multcompare da Statistics Toolbox do Matlab 7.14. Um modelo de regressão (Eq. 1) foi ajustado e analisado com a função LinearModel do mesmo software.

$$
\begin{aligned}
\rho_{\text {bás }}= & \beta_{0}+\beta_{1} \cdot \mathrm{DAP}_{\mathrm{sc}}+\beta_{2} \cdot \mathrm{h}_{\text {total }}+\beta_{3} \cdot \mathrm{h}+\beta_{4} \cdot \mathrm{Dist}+ \\
& \beta_{5} \cdot \mathrm{DAP}_{\mathrm{sc}} \cdot \mathrm{h}_{\text {total }}+\beta_{6} \cdot \mathrm{DAP}_{\mathrm{sc}} \cdot \mathrm{h}+\beta_{7} \cdot \mathrm{DAP}_{\mathrm{sc}} . \\
& \text { Dist }+\beta_{8} \cdot \mathrm{h}_{\text {total }} \cdot \mathrm{h}+\beta_{9} \cdot \mathrm{h}_{\text {total }} \cdot \operatorname{Dist}+\beta_{10} \cdot \mathrm{h} \cdot \\
& \text { Dist }+\beta_{11} \cdot \mathrm{DAP}_{\mathrm{sc}}^{2}+\beta_{12} \cdot \mathrm{h}_{\text {total }}^{2}+\beta_{13} \cdot h^{2}+\beta_{14} \cdot \\
& \text { Dist }^{2}
\end{aligned}
$$

Sendo $\rho_{\text {bás }}=$ massa específica básica $\left(\mathrm{g} / \mathrm{cm}^{3}\right) ; \rho_{\mathrm{n}}=$ coeficientes do modelo; $\mathrm{DAP}_{\mathrm{sc}}=$ diâmetro à altura do peito sem casca $(\mathrm{cm}) ; \mathrm{h}_{\text {total }}=$ altura total $(\mathrm{m}) ; \mathrm{h}=$ posição vertical ou distância a partir da base (m); Dist = posição radial ou distância a partir da medula $(\mathrm{cm})$.

\section{Resultados e Discussão}

\section{Massa específica por árvore}

As diferenças nas médias da massa específica entre as árvores foram significativas estatisticamente (Tabela 2). A amplitude entre as médias foi de $0,075 \mathrm{~g} / \mathrm{cm}^{3}$; o desvio padrão em cada árvore variou de 0,0292 a $0,0585 \mathrm{~g} / \mathrm{cm}^{3}$ e o coefici-

Tabela 1. Diâmetro à altura do peito sem casca $\left(\mathrm{DAP}_{\mathrm{sc}}\right)$, altura total $(\mathrm{ht})$ e altura de retirada dos discos (hd) das árvores amostradas.

Table 1. Diameter at breast height without bark (DAP $\mathrm{sc}_{\mathrm{sc}}$ ), total height $(\mathrm{ht})$ and height of removed slices (hd) from sampled trees.

\begin{tabular}{ccccccc}
\hline Árvore & $\mathrm{DAP}_{\mathrm{sc}}(\mathrm{cm})$ & $\mathrm{ht}(\mathrm{m})$ & \multicolumn{3}{c}{$\mathrm{hd}(\mathrm{m})$} \\
\hline 1 & 29,65 & 20,16 & 3,00 & 6,00 & 9,00 \\
2 & 26,05 & 20,44 & 0,10 & 4,00 & 8,00 & 10,00 \\
3 & 32,45 & 24,37 & 0,10 & 3,00 & 6,00 & 13,00 \\
4 & 56,00 & 27,30 & 5,00 & 9,00 & 12,55 \\
5 & 38,70 & 20,49 & 0,30 & 8,70 & 15,44 \\
6 & 29,40 & 21,55 & 0,30 & 6,30 & 10,35 \\
7 & 33,70 & 19,70 & 0,10 & 6,20 & 11,63 \\
8 & 41,00 & 24,15 & 0,55 & 6,55 & \\
\hline
\end{tabular}


ente de variação apresentou o intervalo de 4,9 a 10\%. Esta diferença significativa entre árvores de uma mesma espécie também foi observada em Abies grandis por Zeidler; Šedivka (2015). A faixa de variação da massa específica básica (Figura 1) está na mesma dos valores apresentados por Brand et al. (2013). Comparativamente, a madeira de eucaliptos relativamente densos como Corymbia torelliana, Eucalyptus resinifera e Eucalyptus cloeziana está na mesma faixa de massa específica (ELEOTÉRIO et al., 2015b).

Os coeficientes de variação observados neste trabalho estão próximos aos apresentados por Kretschmann (2010), de
10\%. Apesar da espécie Miconia cinnamomifolia ser classificada como de rápido crescimento, ela possui madeira mais densa e homogênea que a produzida por Pinus elliottii (MELO et al., 2013), Pinus taeda (MELO, 2015) e Eucalyptus saligna (OLIVEIRA; SILVA, 2003), podendo atender demandas não satisfeitas pela madeira dessas espécies exóticas.

Um modelo linear múltiplo foi ajustado com o objetivo de estimar a massa específica em função das dimensões das árvores e da posição longitudinal e radial. Os coeficientes são apresentados na Tabela 3.

Tabela 2. Número de amostras $(\mathrm{N})$, massa específica média ( $\left.\rho_{\text {bás }}\right)$, desvio padrão $(S)$ e coeficiente de variação $(C V)$.

Table 2. Number of samples $(N)$, average basic density $\left(\rho_{\text {bás }}\right)$, standard deviation $(S)$ and coefficient of variation $(C V)$

$\begin{array}{ccccc}\text { Árvore } & \mathrm{N} & \rho_{\text {bás }}\left(\mathrm{g} / \mathrm{cm}^{3}\right) & \mathrm{S}\left(\mathrm{g} / \mathrm{cm}^{3}\right) & \mathrm{CV}(\%) \\ 5 & 134 & 0,539 \mathrm{e} & 0,0585 & 10,9 \\ 9 & 73 & 0,543 \mathrm{e} & 0,0507 & 9,3 \\ 3 & 186 & 0,572 \mathrm{~d} & 0,0492 & 8,6 \\ 6 & 111 & 0,574 \mathrm{~cd} & 0,0438 & 7,6 \\ 1 & 104 & 0,592 \mathrm{bcd} & 0,0292 & 4,9 \\ 4 & 159 & 0,592 \mathrm{bc} & 0,0471 & 8,0 \\ 8 & 145 & 0,592 \mathrm{bc} & 0,0440 & 7,4 \\ 7 & 152 & 0,597 \mathrm{ab} & 0,0414 & 6,9 \\ & 121 & 0,614 \mathrm{a} & 0,0465 & 7,6 \\ \end{array}$

Médias seguidas pelas mesmas letras não diferem significativamente pelo Teste de Tukey (a=5\%).

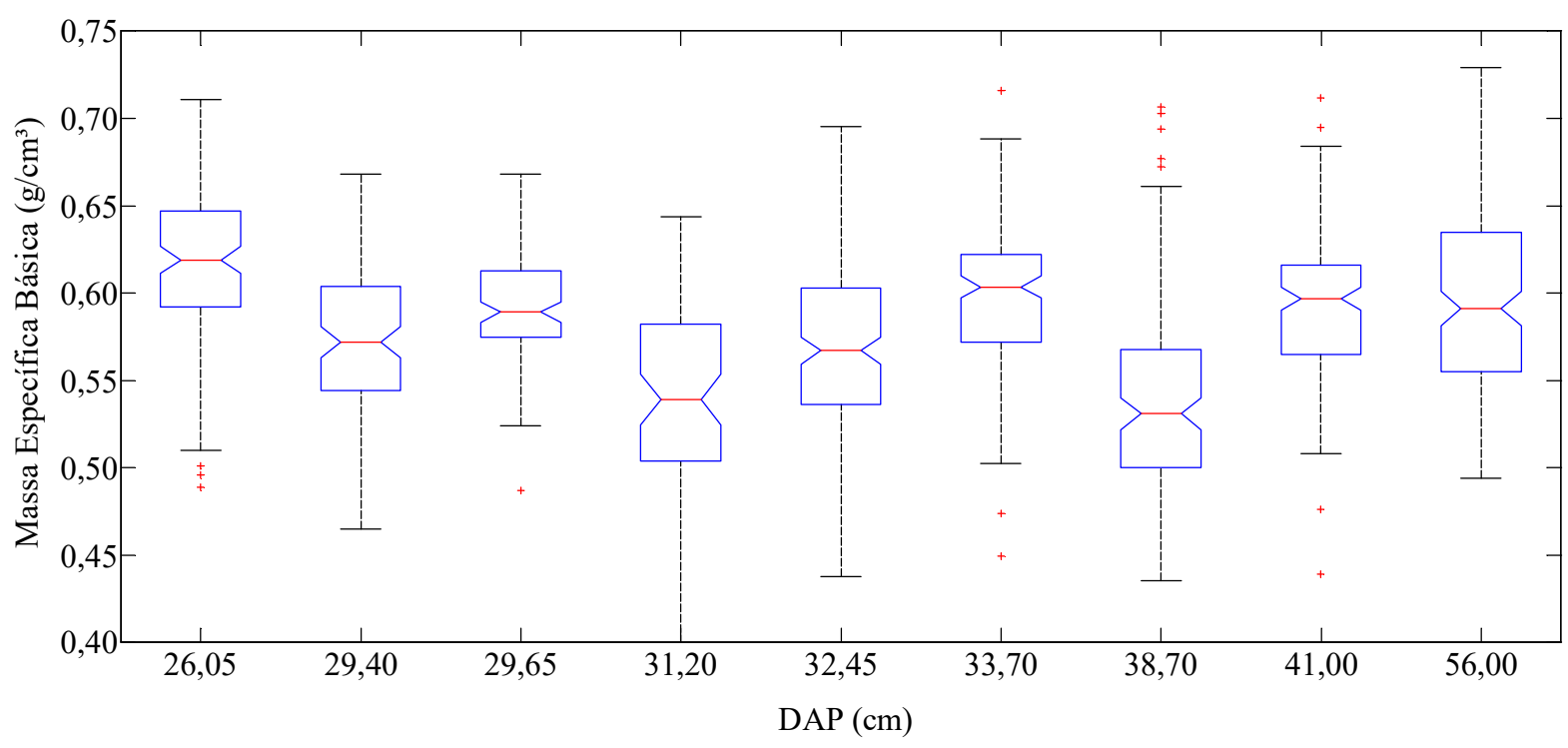

Figura 1. Boxplot da massa específica ordenados pelo $\mathrm{DAP}_{\mathrm{sc}}$ da árvore.

Figure 1. Boxplot of basic density ordered by DHB per tree. 
$\mathrm{O}$ modelo apresentou $\mathrm{R}_{\mathrm{aj}}{ }^{2}=40,3 \%$, teste $\mathrm{F}$ de $58,1\left(\mathrm{P}=2,6^{*} 10^{-123}\right)$ e raiz quadrada do erro médio de 0,039661 .

Na Figura 2 se observam resíduos bem distribuídos, sem tendência, com concentração em torno de $\pm 20 \%$ do valor estimado, sem indícios de heterocedasticidade.

Mäkinen; Hynynen (2012) ajustaram para Pinus sylvestris, modelos com $\mathrm{R}^{2}$ de $36 \%$ para a massa específica em função da idade e da distância da medula. Em Abies grandis a massa específica apresentou $\mathrm{R}^{2}$ de 28,8 com a posição radial e de
8,3\% com a posição vertical, além de diversas interações significativas (ZEIDLER; ŠEDIVKA, 2015).

Além da diferença significativa entre árvores apresentada na Tabela 2, o modelo comprova a complexidade da distribuição da massa específica ao longo dos eixos axial e radial, com efeito significativo do $\mathrm{DAP}_{\mathrm{sc}}$, altura total, altura a partir da base e distância a partir da medula (Figura 3). De maneira geral, a massa específica decresce com o aumento do $\mathrm{DAP}_{\mathrm{sc}}$ e com a distância a partir da medula e cresce com a

Tabela 3. Termos e coeficientes do modelo de regressão, com respectivos erros padrão, valor do Teste $t$ de Student $e$ probabilidade de erro.

Table 3. Terms and coefficients of the regression model, with standard error, $t$ test value and probability of error.

\begin{tabular}{|c|c|c|c|c|c|}
\hline \multirow[t]{2}{*}{ Variável } & \multicolumn{2}{|c|}{ Coeficientes } & \multirow{2}{*}{$\begin{array}{c}\text { Erro Padrão } \\
0,20755\end{array}$} & \multirow{2}{*}{$\begin{array}{l}\text { Teste } t \\
1,0667\end{array}$} & \multirow{2}{*}{$\begin{array}{c}\mathrm{P} \\
0,28635\end{array}$} \\
\hline & $\beta_{0}$ & 0,22138000 & & & \\
\hline $\mathrm{DAP}_{\mathrm{sc}}$ & $\beta_{1}$ & $-0,02515000$ & 0,00263 & $-9,5460$ & $7,57^{\star} 10^{-21}$ \\
\hline $\mathrm{h}_{\text {total }}$ & $\beta_{2}$ & 0,08149300 & 0,02047 & 3,9809 & $7,29^{\star} 10^{-05}$ \\
\hline $\mathrm{h}$ & $\beta_{3}$ & $-0,01330400$ & 0,00285 & $-4,6748$ & $3,28^{\star} 10^{-06}$ \\
\hline Dist & $\beta_{4}$ & $-0,01829200$ & 0,00224 & $-8,1857$ & $7,01^{\star} 10^{-16}$ \\
\hline $\mathrm{DAP}_{\mathrm{sc}}{ }^{\star} \mathrm{h}_{\text {total }}$ & $\beta_{5}$ & 0,00247260 & 0,00027 & 9,1588 & $2,28^{\star} 10^{-19}$ \\
\hline $\mathrm{DAP}_{\mathrm{sc}}^{\star} \mathrm{h}$ & $\beta_{6}$ & 0,00018594 & $5,32^{\star} 10^{-5}$ & 3,4977 & 0,000487 \\
\hline $\mathrm{DAP}_{\mathrm{sc}}{ }^{*}$ Dist & $\beta_{7}$ & 0,00022121 & $4,63^{\star} 10^{-5}$ & 4,7810 & $1,97^{\star} 10^{-06}$ \\
\hline $\mathrm{h}_{\text {total }}{ } \mathrm{h}$ & $\beta_{8}$ & 0,00079337 & 0,00017 & 4,5398 & $6,21^{\star} 10^{-06}$ \\
\hline $\mathrm{h}_{\text {total }}{ }^{\star}$ Dist & $\beta_{9}$ & 0,00078593 & 0,00014 & 5,5625 & $3,29^{\star} 10^{-08}$ \\
\hline $\mathrm{h}^{\star}$ Dist & $\beta_{10}$ & $-0,00050234$ & $7,39^{\star} 10^{-5}$ & $-6,8001$ & $1,66^{\star} 10^{-11}$ \\
\hline $\mathrm{DAP}_{\mathrm{sc}}^{2}$ & $\beta_{12}$ & $-0,00047292$ & $6,41^{\star} 10^{-5}$ & $-7,3760$ & $3,08^{\star} 10^{-13}$ \\
\hline $\mathrm{h}_{\text {total }}^{2}$ & $\beta_{13}$ & $-0,00390730$ & 0,00052 & $-7,5740$ & $7,32^{\star} 10^{-14}$ \\
\hline $\mathrm{h}^{2}$ & $\beta_{14}$ & $-0,00045392$ & $6,74^{\star} 10^{-5}$ & $-6,7315$ & $2,62^{\star} 10^{-11}$ \\
\hline Dist $^{2}$ & $\beta_{15}$ & $-0,00050106$ & $4,82^{\star} 10^{-5}$ & $-10,392$ & $2,96^{*} 10^{-24}$ \\
\hline
\end{tabular}
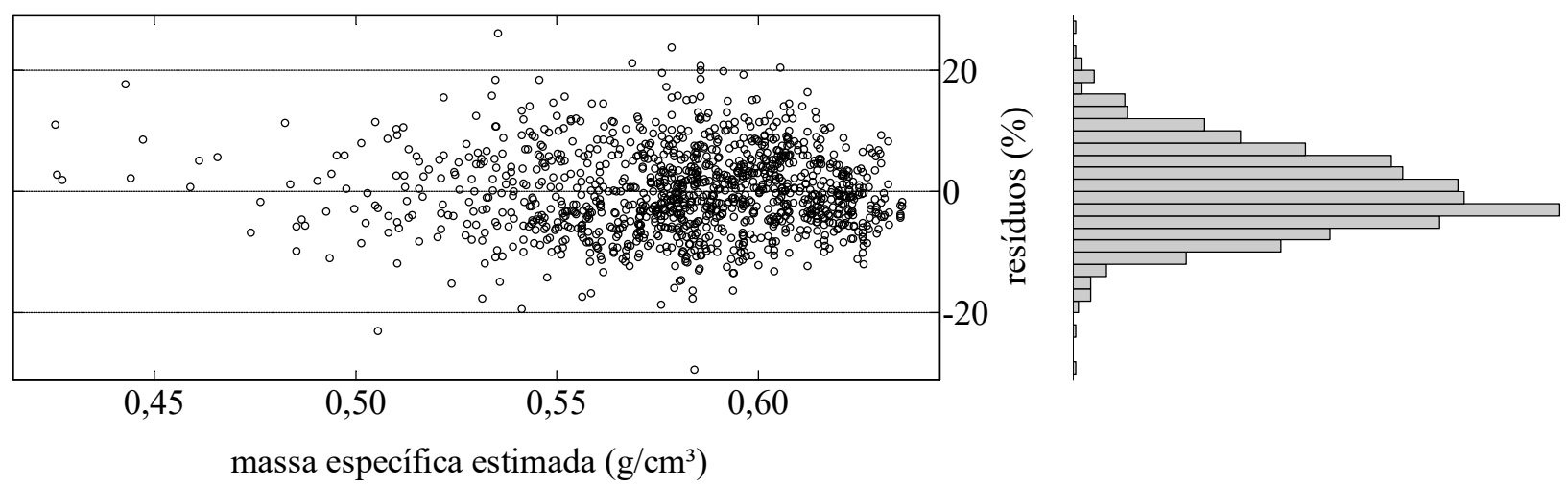

massa específica estimada $\left(\mathrm{g} / \mathrm{cm}^{3}\right)$

Figura 2. Resíduos e histograma dos resíduos relativos do modelo de regressão em função da massa específica estimada.

Figure 2. Residuals and histogram of regression model relative residuals as a function of estimated basic density. 
altura a partir da base e com a altura total da árvore, embora esses dois últimos fatores tenham efeitos menores. Essas considerações não podem ser generalizadas, pois foram encontradas numerosas e significativas interações entre os fatores (Tabela 3).

Valores de massa específica decrescendo com a proximidade da casca são mais raros e foram observados em Quercus faginea (SOUSA et al., 2016), em Quercus suber (KNAPIC et al., 2008) e em Quercus petraea (WASSENBERG et al., 2015, LONGUETAUD et al., 2016). Este padrão é comum em espécies secundárias tardias, que apresentam massa específica mais alta e menor variação radial que as secundárias iniciais. Uma hipótese para a menor massa específica encontrada nas porções mais externas pode ser a presença de alburno, que em Miconia cinnamomifolia é indistinto (CARVALHO, 2006). Como no alburno não há o acúmulo de extrativos que ocorre no cerne, essa porção apresenta menor massa específica, hipótese também considerada por Fajardo (2016) para Nothofagus betuloides e Nothofagus pumilio. Outra hipótese seria a alocação de material lignocelulósico em outras porções da árvore, como em galhos, que na espécie aqui investigada representam um terço da biomassa acima do solo (informação verbal) ${ }^{1}$. A mudança na alocação da biomassa, do tronco para a copa, se justificaria pelo maior diâmetro da árvore, que impacta em maior rigidez do tronco, não sendo tão necessária a densificação.

O efeito positivo da altura ao longo do fuste na massa específica básica pode ser explicado pela necessidade de fortalecimento das porções próximas ao ponto de inversão morfológica, o que também foi observado em outras espécies folhosas, como Hovenia dulcis (ELEOTÉRIO et al., 2015a).

Apesar de existirem algumas informações sobre o histórico de uso da área de estudo, não foi possível obter dados exatos da idade das árvores. De acordo com Palermo et al. (2003), o aumento da espessura da parede celular e a diminuição da largura das células com o avanço da idade induzem também o aumento da massa específica. No entanto, neste estudo não foi possível relacionar a idade com seus possíveis efeitos sobre a massa específica.

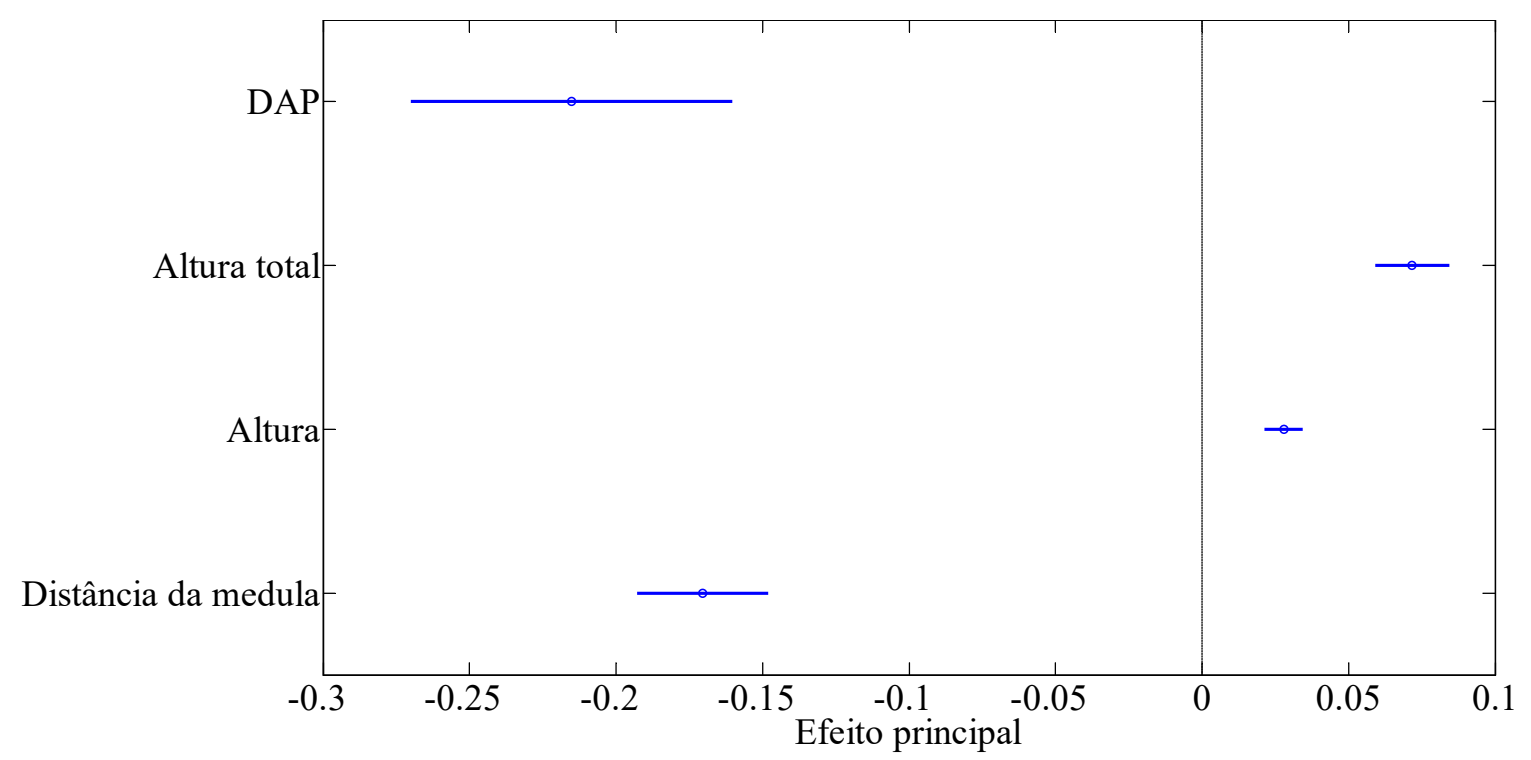

Figura 3. Efeito dos fatores significativos sobre a massa específica média.

Figure 3. Effect of significant factors on average basic density.

1 Informação fornecida por Aline Renata Klitzke.

Departamento de Engenharia Florestal, FURB, Blumenau -

SC), em junho de 2017. 
A Figura 4 exemplifica a estimativa da massa específica em diferentes alturas, até o ponto de inversão morfológica, na árvore 1, com $\mathrm{DAP}_{\mathrm{sc}}$ de $29,65 \mathrm{~cm}$ e altura total de $20,16 \mathrm{~m}$. Para essa árvore observa-se aumento na massa específica ao longo da altura e redução à medida que distancia da medula.

Para Miconia cinnamomifolia, a massa específica não é um bom indicador da transição entre lenho juvenil e adulto. A maior massa específica nas porções mais jovens pode ser uma estratégia da planta para compensar o maior ângulo microfibrilar e a consequente menor resistência mecânica. Entretanto, esse aspecto, bem como a possível relação positiva entre a maior concentração de extrativos no cerne e a massa específica são hipóteses que necessitam de comprovação.

\section{Conclusões}

A massa específica básica média da Miconia cinnamomifolia é de $0,581 \mathrm{~g} / \mathrm{cm}^{3}$, com diferença significativa entre árvores.

Há variações significativas ao longo dos eixos longitudinal e radial das árvores, com efeitos positivos em função da altura em que a massa específica é determinada e da altura total da árvore.

$\mathrm{O} \mathrm{DAP}_{\mathrm{sc}}$ e a posição no sentido radial têm efeito negativo na massa específica.

O modelo de regressão obtido é altamente significativo e permite estimar a massa específica básica em função das dimensões da árvore e da posição dentro no tronco, entretanto tem coeficiente de determinação moderado.

\section{Agradecimentos}

A Food and Agriculture Organization pelo auxílio financeiro através do projeto GCP/BRA/079/GFF. Ao Prof. Dr. Alfredo Celso Fantini, como coordenador do projeto "Ecologia do Ecossistema e Produção Sustentável de Madeira em Florestas Secundárias da Mata Atlântica”. Ao Conselho Nacional de Pesquisa (CNPq) pela bolsa de produtividade em pesquisa do segundo autor. Aos Srs. Clemente Bisewski e Cristiano Bisewski pelo acesso à área e auxílio na coleta do material. Ao Inventário Florístico Florestal do Estado de Santa Catarina

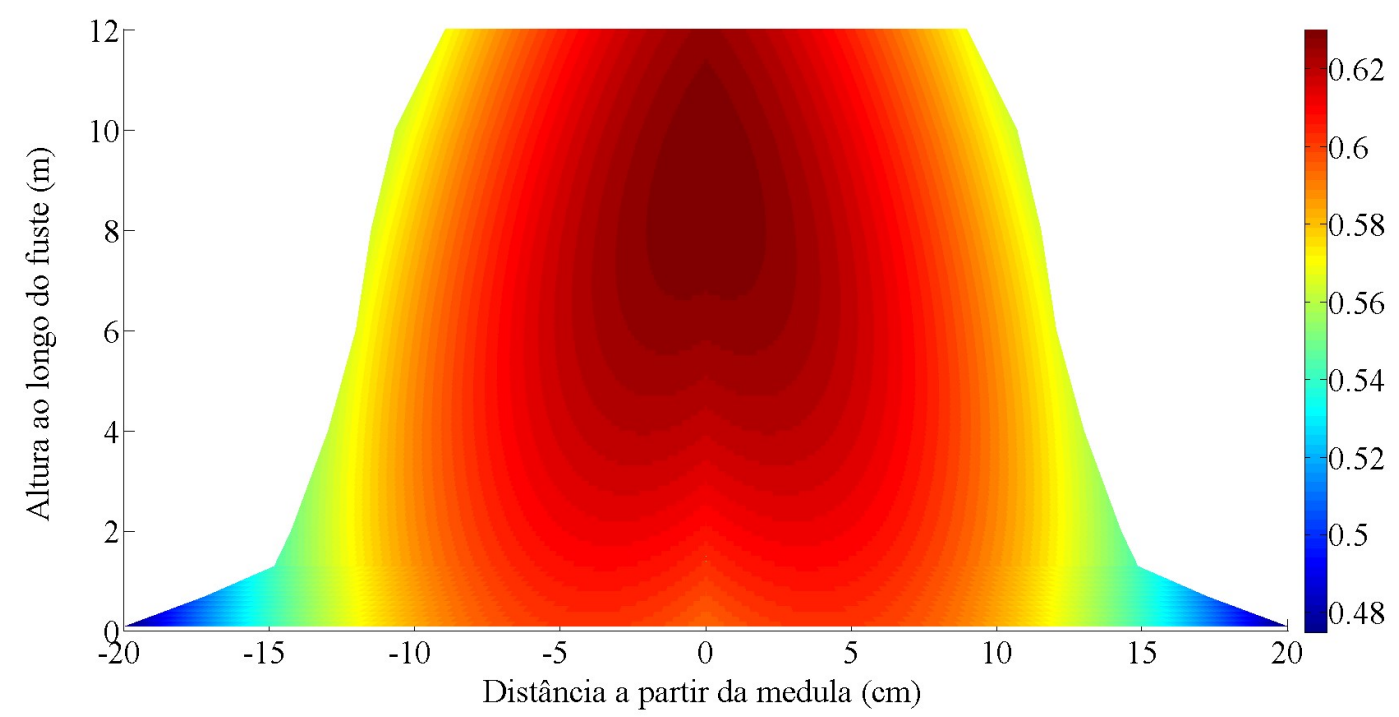

Figura 4. Perfil da massa específica básica estimada ao longo da altura e do diâmetro de uma árvore de Miconia cinnamomifolia com $\mathrm{DAP}_{\text {sc }}$ de $29,65 \mathrm{~cm}$ e altura total de $20 \mathrm{~m}$, de acordo com o modelo e com os coeficientes apresentados na Equação 1 e Tabela 3, respectivamente.

Figure 4: Density profile estimated along height and diameter of Miconia cinnamomifolia tree with DHB of 29,65 cm and total height of $20 \mathrm{~m}$, in accordance with de model and coefficients showed in Equation 1 and Table 3, respectively. 
pelo compartilhamento de informações.

\section{Referências}

ALVARES, C.A.; STAPE, J.L.; SENTELHAS, P.C.; GONÇALVES, J.L.M.; SPAROVEK, G. Köppen's climate classification map for Brazil. Meteorologische Zeitschrift, v.22, n.6, p.711-728, 2013.

BASTIN, J.; FAYOLLE, A.; TARELKIN, Y.; BULCKE, J.V.; HAULLEVILLE, T.; MORTIER, F.; BEECKMAN, H.; ACKER, J.; SERCKX, A.; BOGAERT, J.; CANNIÈRE, C. Wood specific gravity variations and biomass of central African tree species: the simple choice of the outer wood. Plos One, v.10, n.11, p.1-16, 2015.

BRAND, M.A.; CUNHA, A.B.; CARVALHO, A.F.; BREHMER, D.R.; KÜSTER, L.D. Análise da qualidade da madeira e do carvão vegetal produzido a partir da espécie Miconia cinnamomifolia (De Candolle) Naudin (Jacatirãoaçu) na agricultura familiar, em Biguaçu, Santa Catarina. Scientia Forestalis, v.41, n.99, p.401-410, 2013.

CARVALHO, P.E.R.. Jacatirão-açú. Colombo: Embrapa, 2006. (Circular técnica 119).

DENG, X.; ZHANG, L.; LEI, P.; XIANG, W.; YAN, W. Variations of wood basic density with tree age and social classes in the axial direction within Pinus massoniana stems in Southern China. Annals of Forest Science, v.71, n.4, p.505-516, 2014.

ELEOTÉRIO, J.R.; PELLENS, G.C.; COMMANDULI, M.J. Variação longitudinal da massa específica da madeira e da casca de Hovenia dulcis Thunberg. Ciência da Madeira, v.6, n.2, p.122-128, 2015.

ELEOTÉRIO, J.R.; REICHERT, D.; HORNBURG, K.F.; MENEGUELLI, I. Massa específica e retratibilidade da madeira de seis espécies de eucalipto cultivadas no litoral de Santa Catarina. Revista Floresta, v.45, n.2, p.329-336, 2015.

EMBRAPA - Empresa Brasileira de Pesquisa Agropecuária. Solos do Estado de Santa Catarina. Rio de Janeiro: Embrapa, 2004. 745 p. (Boletim de Pesquisa e Desenvolvimento 46).

FAJARDO, A. Wood density is a poor predictor of competitive ability among individuals of the same species. Forest Ecology and Management, v.372, p.217-225, 2016.
FANTINI, A. C.; SIMINSKI, A.; GAIO, M. F. Madeira de florestas secundárias em SC: Nova fonte de renda para os agricultores. Agropecuária Catarinense, v.29, n.2, p.15-18, 2016.

FANTINI, A.C.; SIMINSKI, A. Recuperação natural da vegetação após uso agrícola da terra no litoral de Santa Catarina. In: Anais do VI Congresso Simpósio Nacional e Congresso Latino Americano de Recuperação de Áreas Degradadas, 2005, Curitiba/PR. 2005. p.147-155.

FERNANDES, C.; GASPAR, M.J.; PIRES, J.; SILVA, M.E.; CARVALHO, A.; BRITO, J.L.; LOUSADA, J.L. Within and between-tree variation of wood density componentes in Pinus sylvestris at five sites in Portugal. European Journal of Wood and Wood Products, v.75, n.4, p.511-526, 2017.

GOLDENBERG, R.; CADDAH, M. K. Miconia. In: Lista de Espécies da Flora do Brasil. Rio de Janeiro: Jardim Botânico do Rio de Janeiro. 2015. Disponível em $<$ http://floradobrasil.jbrj.gov.br/jabot/floradobrasil/FB9698> . Acesso em 10 de maio de 2016.

GOUSSANOU, C.A., GUENDEHOU, S., ASSOGBADJO, A.E., KAIRE, M., SINSIN B., CUNI-SANCHEZ, A. Specific and generic stem biomass and volume models of tree species in a West African tropical semi-deciduous forest. Silva Fennica, v.50, n.2, p.1-32, 2016.

GUILLEY, E.; HERVÉ, J.C.; NEPVEU, G. The influence of site quality, silviculture and region on wood density mixed model in Quercus petraea Liebl. Forest Ecology and Management, v.189, n.1, p.111-121, 2004.

IBGE - Instituto Brasileiro de Geografia e Estatística. Manual Técnico da Vegetação Brasileira. $2^{\mathrm{a} e d}$ revisada e ampliada. 2012. 271p

KLEIN, R. M. Mapa Fitogeográfico do Estado de Santa Catarina. Itajaí: HBR, 1978. 24 p.

KNAPIC, S.; LOUZADA, J.L.; LEAL, S.; PEREIRA, H. Within-tree and between-tree variation of wood density components in cork oak trees in two sites in Portugal. Forestry, v.81, n.4, p.465-473, 2008.

KRETSCHMANN, D.E. Mechanical properties of wood. In: Forest Products Laboratory. Wood handbook: Wood as an engineering material. Madison: USDA, Forest Service, Forest Products Laboratory. p. 1-46. 2010. 
LONGUETAUD, F.; MOTHE, F.; FOURNIER, M.; DLOUHA, J.; SANTENOISE, P.; DELEUZE, C. Within-stem maps of wood density and water content for characterization of species: a case study on three hardwood and two softwood species. Annals of Forest Science, v.73, p.601-614, 2016.

MÄKINEN, H.; HYNYNEN, J. Predicting wood and tracheid properties of Scots pine. Forest Ecology and Management, v.279, p.11-20, 2012.

MATE, R.; JOHANSSON, T.; SITOE, A. Biomass equations for tropical forest tree species in Mozambique. Forests, v.5, n.3, p.535-556, 2014.

MELO, R.R. Radial and longitudinal variation of Pinus taeda L. wood basic density in different ages. Revista de Ciências Agrárias, v.58, n.2, p.192-197, 2015.

MELO, R.R. Variação radial e longitudinal da densidade básica da madeira de Pinus elliottii Engelm. com diferentes idades. Ciência da Madeira, v.4, n.1, p.1-11, 2013.

NOCK, C.A.; GEIHOFER, D.; GRABNER, M.; BAKER, J.P.; BUNYAVEJCHEWIN, S.; HIETZ, P. Wood density and its radial variation in six canopy tree species differing in shadetolerance in western Thailand. Annals Of Botany, v.104, n.2, p.297-306, 2009.

OLIVEIRA, J.T.S.; SILVA, J.C. Variação radial da retratibilidade e densidade básica da madeira de Eucalyptus saligna Sm. Revista Árvore, v.27, n.3, p.381-385, 2003.

OLIVEIRA, J.T.S.; SILVA, J.C.; Variação radial da retratibilidade e densidade básica da madeira de Eucalyptus saligna Sm. Revista Árvore, v.27, n.3, p.381-385, 2003.

OLIVEIRA-FILHO A.T. Um sistema de classificação fisionômico-ecológico da vegetação neotropical: segunda aproximação. In: Eisenlohr P. V., Felfili J. M., Melo M. M. R. F., Andrade L. A., Meira Neto J. A. A. (eds) Fitossociologia no Brasil: métodos e estudos de casos, Viçosa, Brasil: Editora UFV, p. 452-473. 2015.

PALERMO, G.P.M.; LATORRACA, J.V.F.; REZENDE, M.A.; NASCIMENTO, A.M.; SEVERO, E.T.D.; ABREU, H.S. Análise da densidade da madeira de Pinus elliottii Engelm por meio de radiação gama de acordo com as direções estruturais (longitudinal e radial) e a idade de crescimento. Floresta e Ambiente, v.10, n.2, p.47-57, 2003.

PELOZZI, M.M.A.; SEVERO, E.T.D.; CALONEGO, F.W.; RODRIGUES, P.L.M. Propriedades físicas dos lenhos juvenil e adulto de Pinus elliottii Engelm var. elliottii e de Eucalyptus grandis Hill ex Maiden. Ciência Florestal, v.22, n.2, p.305$313,2012$.

RAMANANANTOANDRO, T.; RAMANAKOTO, M.F.; RAJOELISON, G.L.; RANDRIAMBOAVONJY, J.C.; RAFIDIMANANTSOA, H.P. Influence of tree species, tree diameter and soil types on wood density and its radial variation in a mid-altitude rainforest in Madagascar. Annals of Forest Science, v.73, n.4, p.1113-1124, 2016.

RAPPAPORT, D.; MONTAGNINI, F. Tree species growth under a rubber (Hevea brasiliensis) plantation: native restoration via enrichment planting in southern Bahia, Brazil. New forests, v.45, n.5, p.715-732, 2014.

REITZ, R; KLEIN, R. M.; REIS, A. Projeto madeira de Santa Catarina. Sellowia, Itajaí, n. 28/30, p. 1-320, 1978.

SCHUCH, C.; SIMINSKI, A.; FANTINI, A. C. Usos e potencial madeireiro do jacatirão-açu (Miconia cinnamomifolia (De Candole) Naudin) no litoral de Santa Catarina. Floresta, v.38, n.4, p.735-741, 2008.

SCHWARTZ, G.; FERREIRA, M.S.; LOPES, J.C. Silvicultural intensification and agroforestry systems in secondary tropical forests: a review. Revista de Ciências Agrárias, v.58, n.3, p.319-326, 2016.

SIAU, John F. Transport processes in wood. Berlin: Springer, 1984.

SILVA, D.A.; KLITZKE, A.R.; ULLER, H.F. Alterações na estrutura fitossociológica do estrato arbóreo causadas pelo corte seletivo em uma área de manejo florestal em Santa Catarina. In: SIMPÓSIO LATINO-AMERICANO SOBRE MANEJO FLORESTAL, 6., 2015, Santa Maria. Anais... Santa Maria: UFSM, CCR, Programa de Pós-Graduação em Engenharia Florestal, 2015. v. 1, p. 77 - 83.

SOTELO MONTES, C.; WEBER J.C.; GARCIA R.A.; SILVA D.A.; MUÑ ${ }^{\sim} I Z$, G.I.B. Variation in growth, wood stiffness and density, and correlations between growth and wood stiffness and density in five tree and shrub species in the Sahelian and Sudanian ecozones of Mali. Trees, v.31, n.3, p.833-849, 2017.

SOUSA, V.B.; LOUZADA, J.L.; PEREIRA, H. Age trends and within-site effects in wood density and radial growth in Quercus faginea mature trees. Forest Systems, v.25, n.1, p.19, 2016. 
VIBRANS, A.C.; MOSER, P.; MAÇANEIRO, J.P. de; LINGNER, D.V.; SILVA, L.S.; PIAZZA, G.A. Amostragem dos remanescentes florestais da Floresta Ombrófila Densa em Santa Catarina. In: VIBRANS, A.C.; SEVEGNANI, L.; GASPER, A.L. de; LINGNER, D.V. (eds). Inventário Florístico Florestal de Santa Catarina. Blumenau: Edifurb, v.4, p 37-95. 2013.

VIDAURRE, G.B.; LOMBARDI, L.R.; OLIVEIRA, J.T.S.; ARANTES, M.D.C. Lenho juvenil e adulto e as propriedades da madeira. Floresta Ambiente, v.18, n.4, p.469-480, 2011.

WASSENBERG, M.; CHIU, H.S.; GUO, W.; SPIECKER, H. Analysis of wood density profiles of tree stems: incorporating vertical variations to optimize wood sampling strategies for density and biomass estimations. Trees, v.29, n.2, p.551-561, 2015.

ZEIDLER, A.; ŠEDIVKA, P. Influence of selected factors on wood density variability in grand fir (Abies grandis/Douglas/Lindl.). Wood Research, v.60, n.1, p.33-44, 2015.

ZOBEL, B.; VAN BUIJTENEN, J.P. Wood variation: its causes and control. Berlin: Springer, 1989. 